\title{
Porpora trombotica trombocitopenica e insufficienza renale
}

\author{
S. Farsetti, C. Somma, L. Caroti, C. Cirami, F. Bergesio, S. Bandini, M. Salvadori
}

S.O.D. Nefrologia dei Trapianti e Dialisi, AOU Careggi, Firenze

Maschio, caucasico, anni 32.

All'età di due anni, in seguito alla comparsa di poliuria, esegue accertamenti medici con riferito riscontro di ipoplasia renale bilaterale, proteinuria e lieve rialzo dei valori di azotemia (in presenza di sCr nella norma). Il paziente riferisce di aver poi effettuato periodici esami ematici ed ecografie addominali, risultati sostanzialmente nella norma fino all'età di 15 anni, quando viene riscontrata lieve insufficienza renale $(\mathrm{sCr} 1.4-1.5 \mathrm{mg} / \mathrm{dL})$. Non ha mai effettuato alcuna terapia specifica.

Durante l'infanzia riferita crisi epilettica, per cui il paziente è stato sottoposto a terapia antiepilettica non specificata per circa un anno. Non sono state effettuate ulteriori indagini diagnostiche.

Quattro giorni prima del ricovero si verifica episodio sincopale con caduta a terra e perdita di coscienza, per cui il paziente viene condotto nel PS dell'ospedale più vicino al suo domicilio. Vengono effettuati TC cranio ed $\mathrm{RX}$ rachide cervicale risultati negativi ed una ecografia dell'addome che rileva reni di dimensioni lievemente ridotte, in particolare il destro; all'ingresso erano presenti anche dolore addominale e nausea. Vengono quindi effettuati esami ematici che evidenziano piastrinopenia $\left(12.000 / \mathrm{mm}^{3}\right)$, anemia $(9.6 \mathrm{~g} / \mathrm{dL})$, aumento delle $\mathrm{LDH}$ (1726 UI/L) e insufficienza renale $(\mathrm{sCr} 2.36 \mathrm{mg} / \mathrm{dL})$. Il paziente viene quindi trattato con emotrasfusione di GR concentrati ed infusione di plasma fresco. Viene trasferito presso il nostro reparto per gli accertamenti e le cure del caso.

All'ingresso vengono effettuati esami ematici e urinari con riscontro di: $\mathrm{Hb} 8.4 \mathrm{~g} / \mathrm{dL}$, piastrine $9000 / \mathrm{mm}^{3}, \mathrm{LDH}$ $822 \mathrm{UI} / \mathrm{L}$, aptoglobina indosabile, schistociti $1 \%$ per campo, test di Coombs diretto e indiretto negativi. I valori di $\mathrm{sCr}$ risultano pari a $2.4 \mathrm{mg} / \mathrm{dL}$ e azotemia pari a 110 $\mathrm{mg} / \mathrm{dL}$. Pur in assenza di una evidente sintomatologia gastroenterica, è stato effettuato esame colturale delle feci risultato negativo per E. Coli 0157 e Shighella.

Nel sospetto di una sindrome emolitico uremica atipica, il paziente inizia trattamento con sedute giornaliere di plasmaferesi e reinfusione di plasma fresco. Vengono inoltre effettuati dosaggio dell'attività dell'ADAMTS 13 (acronimo per "a disintegrin-like and metalloprotease with thrombospondin-1-like domains") e dei suoi inibitori e valutazione multimeri del Fattore di vWillebrand. Inoltre vengono richieste, a completamento diagnostico, una RMN encefalo ed un EEG.

Dopo una iniziale risposta positiva al trattamento con plasmaferesi, evidenziata dal miglioramento del quadro anemico ( $\mathrm{Hb} 10.3 \mathrm{~g} / \mathrm{dL}$ ) e piastrinopenico (fino a 32000 piastrine $/ \mathrm{mm}^{3}$ ), si assiste nuovamente ad una graduale riduzione dei livelli piastrinici, associata a livelli di aptoglobina costantemente indosabili e lieve incremento dell'LDH. A questo punto, data la gravità del quadro clinico e la non responsività al trattamento con plasma, pur non essendo ancora disponibili i risultati dei test richiesti, si intraprende terapia steroidea con Metilprednisolone $40 \mathrm{mg} /$ die e.v., in associazione alle sedute giornaliere di plasma exchange. Dopo circa tre giorni di terapia con steroidi, si assiste ad un progressivo aumento delle piastrine fino al raggiungimento di valori nel range della norma; l'Hb si stabilizza intorno a valori di $12 \mathrm{~g} / \mathrm{dL}$, l'LDH si riduce progressivamente, e i livelli di aptoglobina tornano normali $(1.6 \mathrm{~g} / \mathrm{L})$, consentendo l'interruzione del trattamento con plasmaferesi (in totale 16 sedute). Dal punto di vista renale, i valori di $\mathrm{sCr}$ si stabilizzano intorno a $1.7 \mathrm{mg} / \mathrm{dL}$, con associata proteinuria pari a circa $3 \mathrm{~g} / 24 \mathrm{~h}$.

Nell'arco di alcuni giorni, giungono i referti dei test emocoagulativi che mostrano aumento dei multimeri di vWillebrand, netta riduzione dell'attività di ADAMTS $13(5 \%)$ (V.N 50-150\%) e aumento degli inibitori dell'ADAMTS 13 (22 U/mL; V.N 0-15 U/mL) confermando la diagnosi di Sindrome di Moschowitz da anticorpi anti ADAMTS 13. A ulteriore supporto della diagnosi, giungono anche i referti della RMN encefalo, che mostra la presenza di diffusi esiti microinfartuali, e dell'EEG, che evidenzia anomalie bilaterali lente in presenza di rallentamento del ritmo posteriore. 


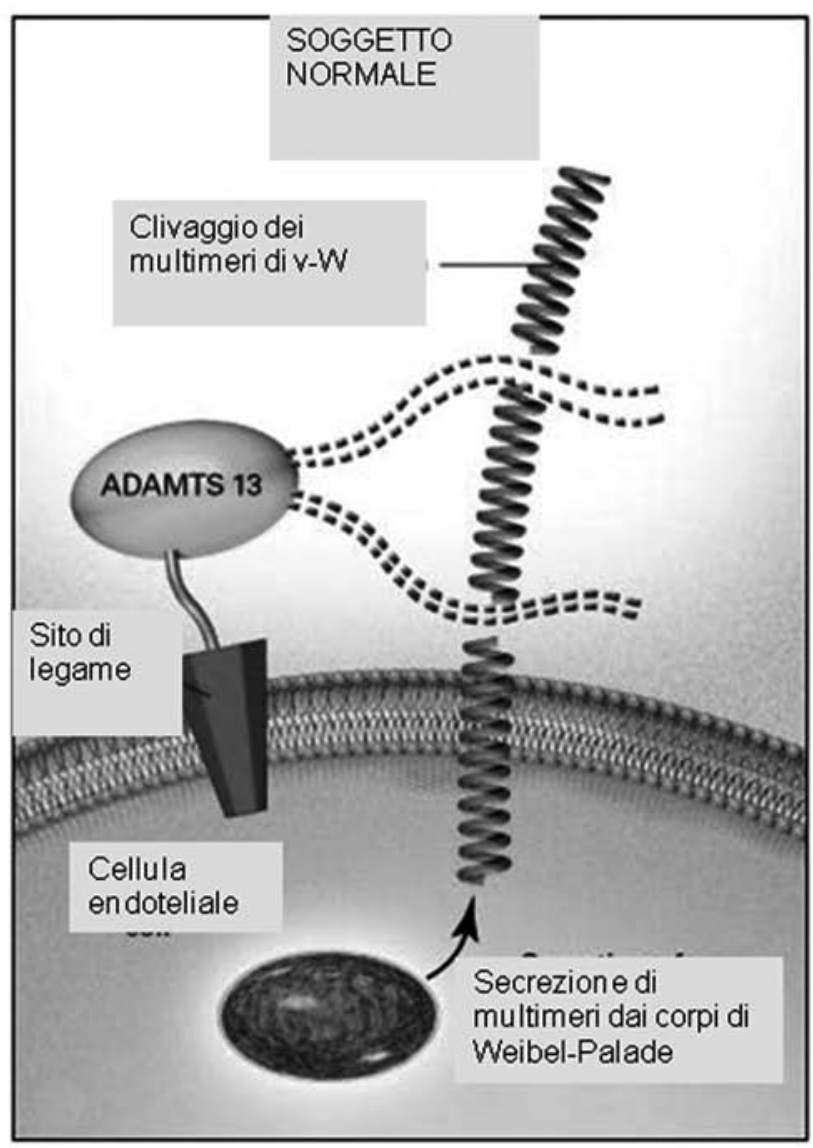

Fig. 1 - Soggetto normale.

Da Moake JL. N Eng J Med 2002; 347 (modificata).

Il paziente prosegue la terapia steroidea con Prednisone $25 \mathrm{mg} \times 2 /$ die per os (circa $0.8 \mathrm{mg} / \mathrm{kg} / \mathrm{die}$ ). Data la stabilità del quadro clinico, viene effettuata agobiopsia renale che all'istologia ottica evidenzia modesto e focale danno tubulo-interstiziale, in assenza di lesioni riconducibili a microangiopatia trombotica.

Dopo la dimissione, il paziente viene seguito presso il nostro DH: il quadro clinico si mantiene stabile, per cui viene progressivamente ridotta la terapia steroidea. Attualmente il paziente assume $5 \mathrm{mg}$ di Prednisone a giorni alterni; l'Hb è pari a circa $14 \mathrm{~g} / \mathrm{dL}$, piastrine $247000 / \mathrm{mm}^{3}$, LDH nella norma, $\mathrm{sCr} 1.7 \mathrm{mg} / \mathrm{dL}$, proteinuria $400 \mathrm{mg} / 24 \mathrm{~h}$.

La Porpora Trombotica Trombocitopenica (PTT o Sindrome di Moschowitz) è una patologia caratterizzata da anemia emolitica microangiopatica, piastrinopenia, porpora, insufficienza renale acuta e importante coinvolgimento neurologico. I pazienti con PTT presentano un deficit congenito o acquisito della degradazione dei multimeri di vWillebrand ad opera di una metalloproteasi plasmatica, denominata ADAMTS 13 (Fig. 1). Il deficit può essere secondario allinibizione della proteasi da

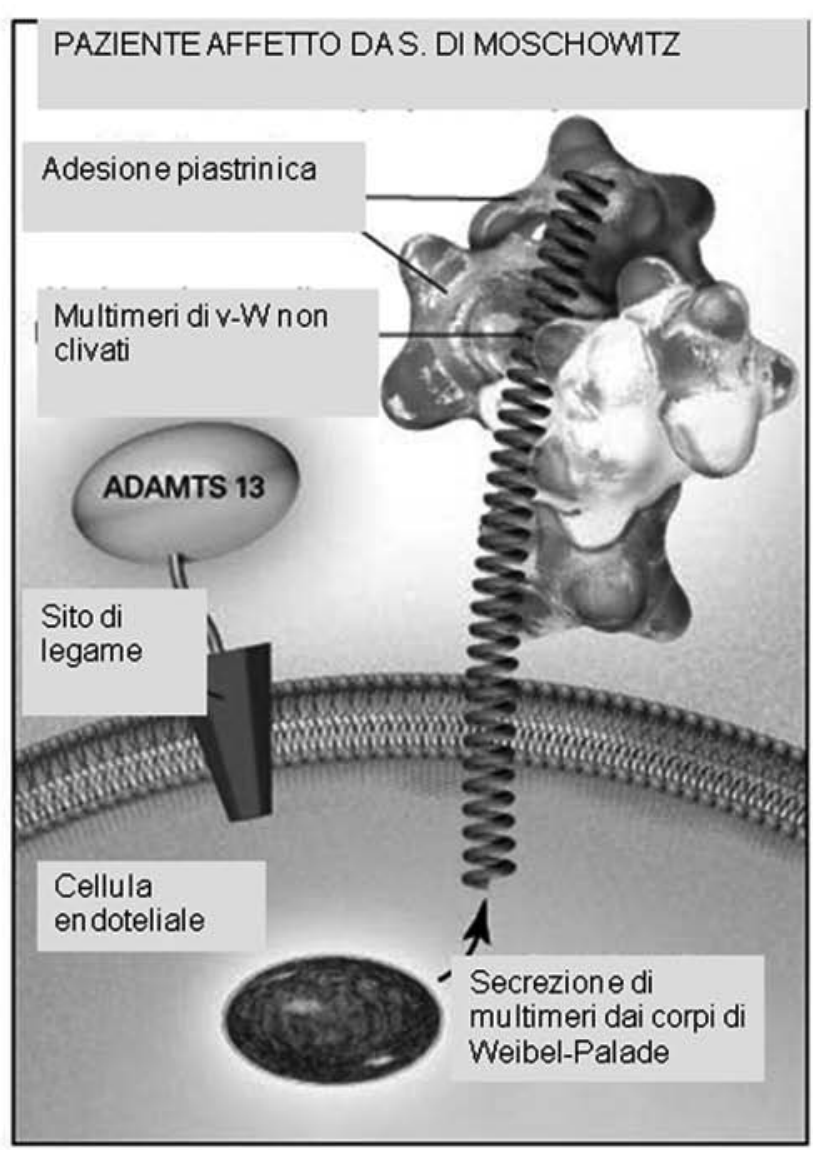

Fig. 2 - Paziente affetto dalla Sindrome di Moschowitz. Da Moake JL. N Eng J Med 2002; 347 (modificata).

parte di anticorpi anti-ADAMTS 13 (forma idiopatica), o a difetti congeniti del gene corrispondente (forma familiare autosomica recessiva). La ridotta $\mathrm{o}$ assente attività della metalloproteasi determina accumulo in circolo dei multimeri di $\mathrm{vW}$ che, legandosi alle piastrine, ne inducono l'aggregazione (Fig. 2). Di conseguenza, si formano trombi piastrinici che occludono il lume dei vasi del microcircolo in vari distretti corporei, prevalentemente a livello renale e cerebrale; si determina quindi anemia emolitica microangiopatica da frammentazione dei globuli rossi, con formazione di schistociti, incremento dell'LDH, consumo di aptoglobina, iperbilirubinemia indiretta, reticolocitosi, piastrinopenia severa. Il test di Coombs è negativo.

Fra le possibili diagnosi differenziali vanno annoverate le altre forme di anemia microangiopatica, ovvero la Sindrome emolitico-uremica tipica (SEU post-infettiva; nel paziente in questione è stato posto il sospetto di SEU post-infettiva in considerazione della sintomatologia gastroenterica presente all'esordio) e quella atipica, nelle forme sporadica (farmaci, post-partum ecc...) e familiare 
(deficit fattori H, I, MCP del complemento). Nella SEU si riscontrano generalmente una riduzione meno severa dei livelli piastrinici, un importante coinvolgimento renale in presenza di un quadro neurologico negativo o lievemente alterato, e un'ipocomplementemia più o meno marcata (in particolare del C3). Inoltre il quadro istologico risulta caratterizzato da ispessimento della parete capillare glomerulare in presenza di trombi nelle arteriole e dei capillari glomerulari, rigonfiamento e distacco dell'endotelio dalla parete dei piccoli vasi, talvolta necrosi glomerulare.

Circa l' $80 \%$ delle PTT idiopatiche risponde al trattamento con plasma exchange, ed il 30\% di esse può presentare recidive della malattia. Il trattamento con plasmaferesi e con infusioni di plasma è quindi consigliato indipendentemente dal riscontro di ridotta attività della metalloproteasi. Tuttavia, in casi resistenti alla terapia con plasmaferesi, il riscontro di inibitori dell'ADAMTS 13 può giustificare l'utilizzo di altri presidi terapeutici, come corticosteroidi, immunosoppressori ed altri immunomodulatori, tra cui l'anticorpo monoclonale anti-CD20 (Rituximab).

\section{Indirizzo degli Autori:}

Calogero Cirami, MD

Nefrologia dei Trapianti e Dialisi

Azienda Ospedaliero-Universitaria-Careggi

Viale Pieraccini 18

Firenze

sandro.bandini1@virgilio.it

\section{TEST DI VERIFICA - 2}

1) Quali farmaci possono essere responsabili di una microangiopatia trombotica con trombi renali o sistemici?
a) Mitomicina
b) Chinino
c) Inibitori della calcineurina
d) Tutti i precedenti
e) Nessuno dei precedenti

2) Nei casi di PTT con croniche recidive è necessario indagare:

a) La presenza di anticorpi anti ADAMTS13

b) Mutazioni genetiche a carico del gene 9q34

c) Ricerca dei multimeri di von Willebrand

d) Tutti i precedenti

3) ADAMTS 13 è:

a) Una metalloproteinasi plasmatica

b) Proteasi calcio e zinco dipendente

c) È un enzima principalmente prodotto dagli epa-

d) $\grave{E}$ un enzima che degrada i multimeri di von

d) Willebrand in monomeri

e) Tutti i precedenti

Le risposte corrette alle domande sono a pag. 51 